\section{Ist sublingual subkutan vergleichbar?}

\author{
Die Überlegenheit der sublingualen Immuntherapie (SLIT) \\ gegenüber Plazebo ist belegt. Jetzt liegen die Ergebnisse des ersten \\ direkten Vergleichs mit dem klassischen Verfahren der subkutanen \\ Immuntherapie (SCIT) vor.
}

D änische Allergologen dokumentierten in einer dreiarmigen randomisierten doppelblinden, mit der Double-Dummy-Technik durchgeführten Studie die Daten von 71 Patienten mit einer durch Birkenpollen induzierten Rhinokonjunktivitis. Die Probanden erhielten entweder eine SCIT (standardisierte, an Kalziumphosphat adsorbierte Birkenpollenextrakte) plus eine Plazebo-SLIT, eine SLIT (standardisierte Birkenpollenextrakte in Tropfenform, die vor dem Herunterschlucken 2 Minuten unter Zunge behalten wurden) plus eine Plazebo-SCIT oder eine Plazebo-SCIT plus eine Plazebo-SLIT. Für eine optimierte Verblindung wurde den PlazeboInjektionslösungen Histamin zugesetzt, das eine Schwellung wie bei SCIT an der Injektionsstelle hervorrufen sollte.

Die Studie dauerte 3 Jahre: Im ersten Jahr wurden die Patienten-Basiswerte während des Pollenfluges erfasst, die beiden nächsten Jahre sollten den Behandlungserfolg zeigen. Aufgrund des sehr schwachen Pollenfluges mit geringen Symptomen im dritten Jahr (1999) wurde nur das erste Behandlungsjahr mit Daten von 58 Patienten ausgewertet.

Im Vergleich zu den Ausgangswerten besserte sich unter der SLIT die mediane Krankheitsschwere auf die Hälfte und unter der SCIT auf ein Drittel, jeweils verglichen mit der Plazebomedikation. Die beiden Verumgruppen unterschieden sich nicht signifikant. Sowohl der Symptom- als auch der Medikationsscore der beiden $\mathrm{Hy}$ posensibilisierungsgruppen zeigte eine statistisch signifikante Verbesserung gegenüber Plazebo. Parameter zur Lebensqualität waren in beiden Behandlungsgruppen vergleichbar. Leichte Nebeneffekte - in der Regel Rhinokonjunktivitis - traten in allen Behandlungsgruppen auf. Reaktionen höherer Schweregrade wurden fünfmal unter
SCIT und einmal unter Plazebo gesehen. Bei SLIT-Patienten traten vor allem in der Auftitrationsphase überproportional häufig Jucken oder ein leichtes Ödem im Mund-Rachen-Raum nach Einnahme der Tropfen auf (13 Fälle unter SLIT, zwei unter SCIT und vier unter Plazebo). Die Lokalreaktio- nen waren durch Dosisreduktion gut beherrschbar.

Fazit: Insgesamt konnte diese Studie eine prinzipielle therapeutische Gleichwertigkeit der SLIT mit der SCIT im ersten Behandlungsjahr zeigen, wobei es nur unter der SCIT zu schwereren systemischen Nebenwirkungen kam. $\quad b k$

Khinchi MS et al. Clinical efficacy of sublingual and subcutaneous birch pollen allergen-specific immunotherapy. Allergy 2004; 59: 45-53

Passalacqua G et al. Sublingual or injection immunotherapy: the final answer? Allergy 2004; 59: 37-8
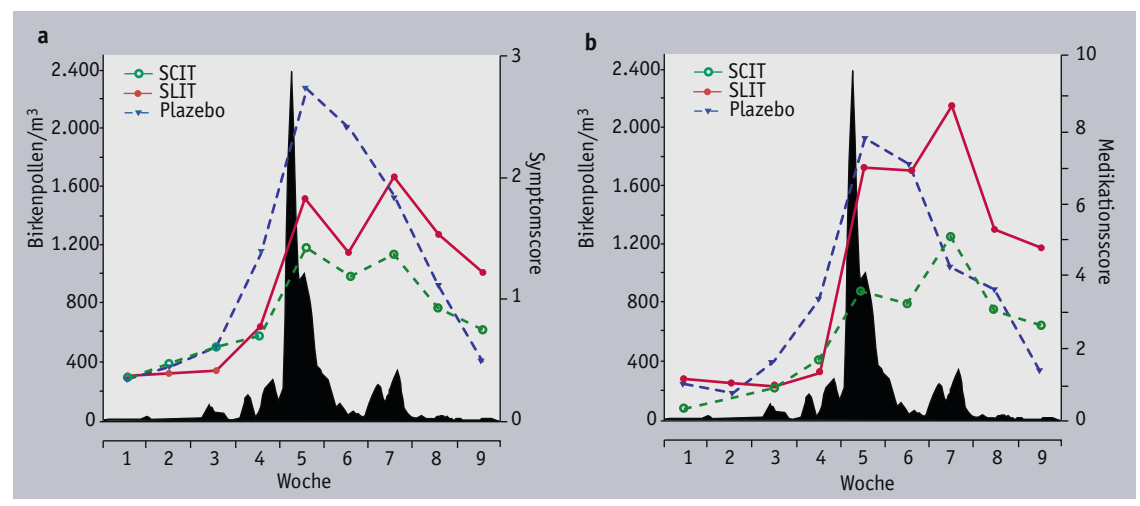

Durchschnittliche wöchentliche Rhinokonjunktivitissymptome (a) und durchschnittlicher wöchentlicher Medikationsscore (b) im zweiten Studienjahr im Vergleich der drei Behandlungsregime. Die schwarze Fläche zeigt die Intensität des Pollenfluges an.

\section{Immuntherapie induziert IgE-Blockade}

\section{Die klinischen Effekte einer spezifischen Hyposensibilisierung} werden unter anderem auf die Ausbildung einer Toleranz gegen die Symptome verursachenden Allergene durch einen „TH2/TH1Switch“ der Zytokinantwort zurückgeführt. Nun richtet sich die Aufmerksamkeit auf die Rolle des Allergen-spezifischen IgG.

$\mathrm{H}$ ohe Spiegel von Allergen-spezifischen IgG-Antikörpern korrelieren negativ mit einer Sensibilisierung, während umgekehrt Atopiker häufig sehr niedrige Allergen-spezifische IgGSerumspiegel aufweisen. Aus zahlreichen Studien ist ein Anstieg der Allergen-spezifischen IgG-Antikörper, speziell des $\mathrm{IgG}_{4}$-Subtyps, infolge einer spezifischen Immuntherapie (SIT) be- kannt. Diese IgG-Antikörper sind in der Lage, die IgE-vermittelte Allergenpräsentation durch B-Zellen an die TZellen zu blockieren. Es wird nun vermutet, dass die Induktion dieser blockierenden Antikörper einen wichtigen Mechanismus der SIT-Schutzwirkung darstellt.

Ermöglicht wurden die aktuellen Forschungen auch durch eine neue 